idea of utility or risk is much more precise than one's beliefs. Any theory of probability is used in order to decide on a course of action, and it would surely be desirable that the consequences of each action be to the fore in any arguments used. For example, the same results will be capable of different interpretations according to the relative utilities of the different actions. Statisticians often try to hide this fact but it is always there, usually expressed in the form of the significance-level used. The omission of such considerations seems to be a flaw of all theories based on 'degrees of belief'. The experimentalist is usually not interested in the 'degrees of belief' he has in different hypotheses, but in the consequence of holding any belief. The frequentist and non-frequentist may argue whether prior probabilities should be used, but surely both should consider the risks, or utilities, as well. The relegation, by the author, of these notions to the 'suggestions' therefore seems misplaced.

The book, the author remarks, may be considered from the philosophical, mathematical or statistical points of view. It is not for me to make any remarks about the first aspect of the theory, beyond those already made. The mathematical development is not complete; it does not pretend to be. Nor is it always rigorous, but this is perhaps excusable since a complete development is available elsewhere, and its inclusion here would only divert attention from the new ideas the author wishes to put forward. The statistical comments are always stimulating, and, although I would not advocate or use the author's method, it must be admitted that many of the methods commonly used, as, for example, the $\chi^{2}$ test, are not based on any very clear foundation and are open to legitimate criticism. There is an excellent summary of the theory, by the author himself, in the discussion of a paper by Barnard ${ }^{3}$.

D. V. LINDLEY

1"Grundbegriffe der Wahrscheinlichkeitsrechnung" (Berlin, 1933). English translation by N. Morrison (Chelsea, N.Y., 1950).

a "Statistical Decision Functions" (Wiley and Sons, New York, 1950). \& J. Roy. Stat. Soc., B, 8, 140 (1949).

\section{FOURIER TRANSFORMS MADE EASY}

Tables of the Function $\sin \varphi / \varphi$ and of its First Eleven Derivatives

By the Staff of the Computation Laboratory. (Annals of the Computation Laboratory of Harvard University, Vol. 22.) Pp. xviii +241 . (Cambridge, Mass. : Harvard University Press ; London : Oxford University Press, 1949.) 63s. net.

7 HESE Tables were computed at the request of the United States Air Force, for use in connexion with research on microwave radiation prob. lems. They will be found specially useful in a large class of problems-radiation, diffraction and statistics are mentioned in an excellent section of the introduction written by Dr. Roy Spencer-where Fourier transforms of functions defined over a finite range, and zero elsewhere, are used and where numerical results are desired. This is due to the fact that $\sin \varphi / \varphi$ is the Fourier transform of a function which is unity in the range between -1 and +1 , and zero elsewhere. Moreover, if the function can be expressed as a polynomial, its Fourier transform involves $\sin \varphi / \varphi$ and its derivatives; while if the function is expressed as a Fourier series, its Fourier transform involves values of $\sin \varphi / \varphi$ at intervals of $\pi$ in $\varphi$.
The Tables give values of the function $g(\phi)=$ $\sin \varphi / \varphi$ and its first eleven derivatives to nine decimals. The argument is in degrees, and the interval is $0.5^{\circ}$. The range is from $0^{\circ}$ to $3,599 \cdot 5^{\circ}$. The twelve values corresponding to any argument are given at a single opening, and the argument is for convenience repeated on the right of the right-hand page ; each page contains thirty arguments. All zeros, decimal points and minus signs are repeated; this makes the pages rather heavy, and may be somewhat fatiguing in use; but presumably it would have been difficult to order the automatic machine, which computed these values and printed its results, to suppress them. Interpolation, when necessary, will most conveniently be done by Taylor series, and coefficients for converting the several terms of this series from the degrees of the argument $\varphi$ to radians are given in the introduction.

The Tables were computed on the, by now wellknown, Harvard machine, and a brief outline of the methods is given in the first section of the introduction. The existence of a very simple relation between successive derivatives made this computation ideally suited to the special aptitudes of this machine.

These Tables give more decimals, smaller interval, greater range, or higher derivatives, than any others of the same functions hitherto published. It is certain that their usefulness will extend to many fields of study and research additional to those already mentioned.

W. G. BICKLEX

\section{ASPECTS OF LUMINESCENCE}

\section{Luminescent Materials}

By G. F. J. Garlick. (Monographs on the Physics and Chemistry of Materials.) Pp. viii +254. (Oxford : Clarendon Press ; London : Oxford University Press, 1949.) 21s. net.

A DOZEN years ago or so the ideas of Wilson on A semi-conductors and the growing realization that the luminescence of solids was probably another part of the same wood led Johnson, Seitz and others to apply the electron energy-band model to the problem. At about the same time, J. T. Randall entered the field already armed with an extensive experience of the 'cookery' of the luminescent materials demanded with increasing insistence by the development of fluorescent lighting and of television. Developing the Urbach thermo-luminescent experiments along with studies of decay-rates, Randall fashioned a powerful weapon for investigating the mechanism of phosphorescence.

It is not surprising, therefore, that Dr. Garlick, joining the team at Birmingham a little later, should have become fired with enthusiasm for the new technique and the implications of its findings, or that his preoccupation with these aspects of the subject should be manifest in this book.

The breezy confidence with which it is written makes for lively reading, but, while bringing down appreciable fruits here and there, leads sometimes to rather forthright assertions with which other workers will not entirely agree. It is, for example, somewhat rash to suggest that luminescence is a property of comparatively few solids and liquids, or that few solids can be said to be luminescent in the pure state. The energy-band model suggests that activating impurities provide possibilities of absorption and emission to and from energy-levels which do not 